\title{
On the Vibration of Single-Walled Carbon Nanocones: Molecular Mechanics Approach versus Molecular Dynamics Simulations
}

\author{
R. Ansari, ${ }^{1}$ A. Momen, ${ }^{1}$ S. Rouhi, ${ }^{2}$ and S. Ajori ${ }^{1}$ \\ ${ }^{1}$ Department of Mechanical Engineering, University of Guilan, P.O. Box 3756, Rasht, Iran \\ ${ }^{2}$ Young Researchers Club, Islamic Azad University, Langroud Branch, P.O. Box 44715-1333, Langroud, Guilan, Iran \\ Correspondence should be addressed to R. Ansari; r_ansari@guilan.ac.ir
}

Received 28 February 2014; Revised 19 May 2014; Accepted 25 May 2014; Published 14 July 2014

Academic Editor: Tony Murmu

Copyright (C) 2014 R. Ansari et al. This is an open access article distributed under the Creative Commons Attribution License, which permits unrestricted use, distribution, and reproduction in any medium, provided the original work is properly cited.

\begin{abstract}
The vibrational behavior of single-walled carbon nanocones is studied using molecular structural method and molecular dynamics simulations. In molecular structural approach, point mass and beam elements are employed to model the carbon atoms and the connecting covalent bonds, respectively. Single-walled carbon nanocones with different apex angles are considered. Besides, the vibrational behavior of nanocones under various types of boundary conditions is studied. Predicted natural frequencies are compared with the existing results in the literature and also with the ones obtained by molecular dynamics simulations. It is found that decreasing apex angle and the length of carbon nanocone results in an increase in the natural frequency. Comparing the vibrational behavior of single-walled carbon nanocones under different boundary conditions shows that the effect of end condition on the natural frequency is more prominent for nanocones with smaller apex angles.
\end{abstract}

\section{Introduction}

Recently, different nanostructures such as carbon nanotubes (CNTs) [1], fullerenes [2], carbon nanorings [3], and carbon nanocones (CNCs) $[4,5]$ have attracted a great deal of researches. Due to diverse potential applications of singlewalled carbon nanocones (SWCNCs) in different areas such as cold electron and field emitter [6], adsorbent [7], and mechanical sensors $[8,9]$, a comprehensive understanding of their mechanical, physical, and electronic properties is necessary.

Researches on CNCs have been started at the same time as CNTs. However, the CNC and its properties attracted less attention. The cones with apex angles of $19.2^{\circ}, 38.9^{\circ}, 60^{\circ}, 84.6^{\circ}$, and $112.9^{\circ}$ were experimentally synthesized by Krishnan et al. [11]. Subsequently, Naess et al. [12] studied the morphologies of the CNCs with different apex angles by using transmission electron microscopy (TEM), synchrotron X-ray, and electron diffraction. Employing a quantum chemical optimization, the accurate geometries of CNCs were determined by Compernolle et al. [13]. It was shown that both armchair and zigzag edge atoms are present simultaneously in the same cone.

Different theoretical approaches have been used to study the properties of nanostructures which can be divided as atomistic and continuum approaches. Molecular dynamics (MD) simulations [14, 15] and density functional theory (DFT) [16] are the most significant approaches of the former approaches, while the latter one can be subdivided to Bernoulli-Euler/Timoshenko beam models [17-19], the shell models [10, 20, 21], the molecular structural models [22-25], and meshless approaches [26-28]. Due to considerable time consumption of the atomistic approaches, continuum methods have attracted more attention. Due to similarity between the molecular structure of nanostructures and a space frame structure, the molecular structural methods have been used to study the mechanical behavior of nanostructures. In these methods, to obtain the physical properties of the model, the energy terms in molecular and structural models are equated. 
As in meshless methods, the problem domain is modeled by using a set of scattered nodes; the common difficulties of mesh usage including inaccurate and unstable solutions caused by distortion of meshes [26] and high costs of remeshing are omitted [27]. So, they can be usefully applied to study the nanostructures. Recently, meshless methods have been used extensively in the investigation of functionally graded panels reinforced by nanostructures [26-28].

Some theoretical studies on mechanical behavior of CNCs can be found in the literature. MD simulation was used by Wei et al. [29] to study the mechanical behavior of CNCs. Liew et al. [30] analyzed the axial buckling of CNCs. Using the beam model and MD simulations, Hu et al. [8] investigated the free transverse vibration of SWCNCs. Using a nonlocal continuum shell model, Firouz-Abadi et al. [31] studied free vibration characteristics of nanocones. They used Galerkin technique to study the influences of geometrical parameters of the nanocone on these frequencies. MD simulations were also employed by Jordan and Crespi [32] to study the mechanical chiral inversion of closed CNCs. An atomically accurate chiral inversion was also obtained in their work, without breaking the chemical bonds. Utilizing the higher order continuum theory, Yan et al. [33] studied the structural parameters and elastic properties of SWCNCs. They employed all five types of CNCs to test the influence of the conical angle on the mechanical properties. A modal analysis of SWCNTs and SWCNCs was performed by J. H. Lee and B. S. Lee [34] employing finite element method (FEM). In their work, natural frequencies and corresponding mode shapes of these nanostructures were calculated. Using FEM, Fakhrabadi et al. [35, 36] predicted vibrational properties and the elastic and buckling behavior of CNCs with different dimensions. They obtained the natural frequencies and corresponding mode shapes as well as the elastic modulus and compressive forces of the axial buckling for CNCs with the various dimensions and boundary conditions.

In this paper, a finite element (FE) model is applied to investigate the vibration behavior of SWCNCs. The effect of boundary condition, apex angle, and length on the fundamental natural frequency of SWCNCs are studied. Besides, MD simulations are used to obtain the natural frequency of SWCNCs. The results of FE model are verified by MD simulations.

\section{Atomic Structure of SWCNCs}

To form a SWCNC, a sector of circular graphene sheet is removed; then the graphene sheet is rolled around the apex and the open edges are joined. Disclination angle $d_{\theta}$, which is defined as the angle of the sector removed from a plain sheet, is utilized to characterize the nanocones. The value of $d_{\theta}$ can only have certain values including $60^{\circ}, 120^{\circ}, 180^{\circ}, 240^{\circ}$, and $300^{\circ}$. Figure 1 displays the sectors of graphene sheet with disclination angles of $60^{\circ}, 120^{\circ}, 180^{\circ}$, and $240^{\circ}$. The apex angle of cones is obtained as $2 \sin ^{-1}\left(1-d_{\theta} / 360\right)$.

Transformation from a graphene sheet with disclination angle $180^{\circ}$ in a polar coordinate system to a three-dimensional cone in Cartesian coordinate system is displayed in Figure 2.
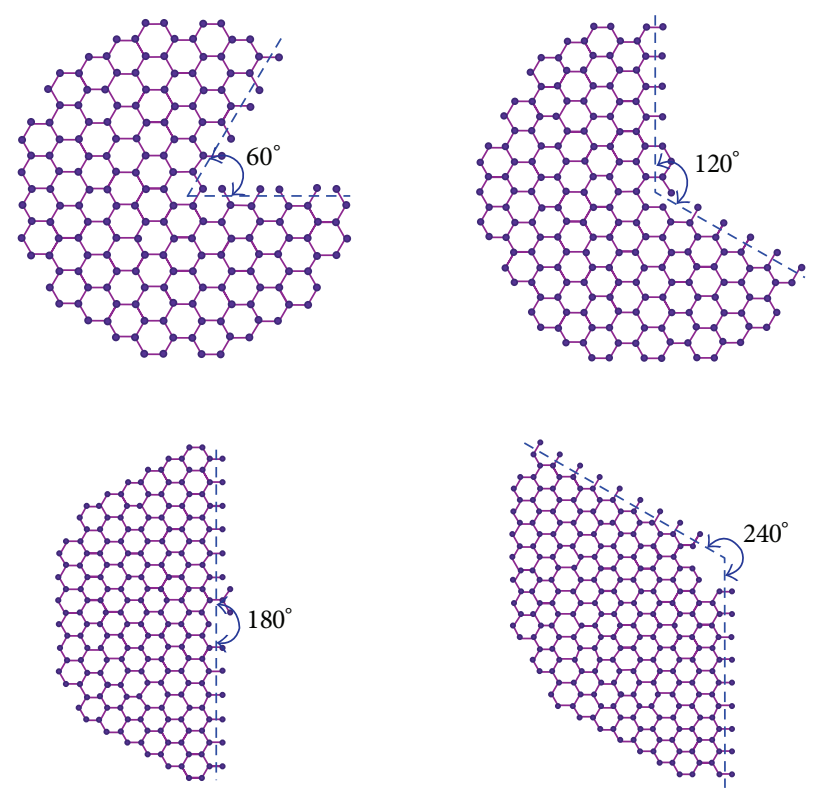

FIGURE 1: Cone sheets with disclination angles of $60^{\circ}, 120^{\circ}, 160^{\circ}$, and $240^{\circ}$.

The polar coordinates of point $P$ in the graphene sector are indicated as $(l, \alpha) ; L$ and $\varphi$ represent the parameters of the graphene sector in which the lengths of $\overline{O A}$ and $\overline{O B}$ are equal. Sector $O A B$ is folded at apex $O$, and $\overline{O A}$ is overlapped by $\overline{O B}$ [20].

Considering the point $P(X, Y, Z)$, the following equations are obtained [34]:

$$
X=r \cos \beta, \quad Y=r \sin \beta, \quad Z=-\sqrt{l^{2}-r^{2}},
$$

in which $\beta$ and $r$ are the unknown variables. The transformation from the graphene sheet to the three-dimensional cone leads to a relation between the corresponding angles as follows:

$$
\frac{\beta}{2 \pi}=\frac{\alpha}{\varphi} .
$$

Rearranging (2) leads to the dihedral angle $\beta$ between $O^{\prime 2} O^{\prime} C^{\prime}$ and $O^{\prime 2} O^{\prime} A^{\prime}$ in the following form:

$$
\beta=\alpha\left(\frac{2 \pi}{\varphi}\right) .
$$

By involving lengths $l$ and $L$, the relation between radii $r$ and $R$ can also be specified as follows:

$$
\frac{r}{R}=\frac{l}{L} .
$$

The base radius of the cone is obtained as follows:

$$
R=\frac{L \varphi}{2 \pi}
$$

Substitution of (5) into (4) gives the radius $r$ as follows:

$$
r=\frac{l}{2 \pi} \text {. }
$$




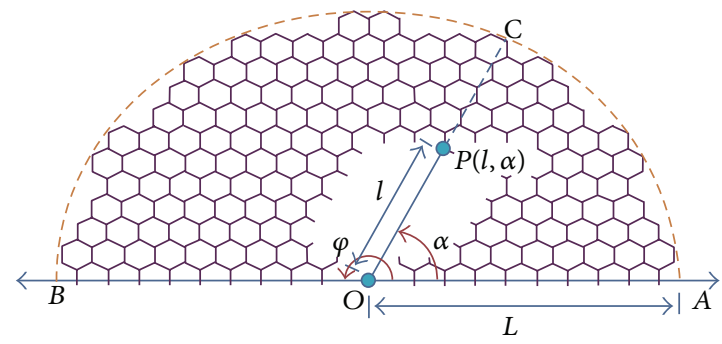

(a)

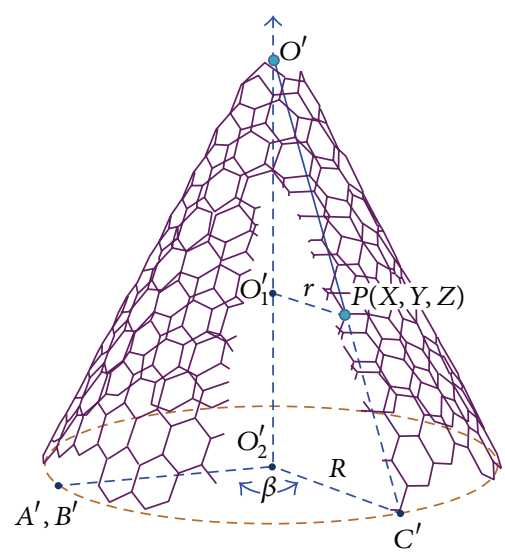

(b)

Figure 2: Symbols and variables used in the transformation from a graphene sheet for a cone $180^{\circ}$.

Substituting (3) and (6) into (1) yields the coordinates of point $P$ in the three-dimensional cone in the following form:

$$
\begin{aligned}
& X=\left(\frac{l \varphi}{2 \pi}\right) \cos \left[\alpha\left(\frac{2 \pi}{\varphi}\right)\right], \\
& Y=\left(\frac{l \varphi}{2 \pi}\right) \sin \left[\alpha\left(\frac{2 \pi}{\varphi}\right)\right], \\
& Z=-l \sqrt{1-\left(\frac{\varphi}{2 \pi}\right)},
\end{aligned}
$$

where $X, Y$, and $Z$ indicate the nanocone atom coordinates, and angles are given in radians.

\section{Molecular Structural Modeling}

The discrete structures of CNCs, as presented in Figure 3, with different lengths and apex angles are utilized in the present finite element modeling. A three-dimensional elastic beam element is employed to model the covalent bonds between the carbon atoms. Carbon atoms are considered as point mass elements. The total potential energy is the summation of energies that emerged from the different interatomic interactions and can be defined as follows [25]:

$$
E=\sum U_{r}+\sum U_{\theta}+\sum U_{\phi}+\sum U_{\omega}+\sum U_{\mathrm{vdW}}
$$

in which $U_{r}, U_{\theta}, U_{\phi}, U_{\omega}$, and $U_{\mathrm{vdW}}$ are energies corresponding to the bond stretching, angle variation, dihedral angle torsion, out-of-plane torsion, and van der Waals (vdW) interactions, respectively. The physical interpretation of these energy terms is given in Figure 4. Due to the small contribution of the vdW interaction in the SWCNC structure, it can be ignored in comparison with the other energies. Discarding the final term of (8), the other terms can be obtained in the forms of

$$
\begin{aligned}
& U_{r}=\frac{1}{2} k_{r}(\Delta r)^{2}, \quad \frac{d^{2} U_{r}}{d \Delta r^{2}}=k_{r}, \\
& U_{\theta}=\frac{1}{2} k_{\theta}(\Delta \theta)^{2}, \quad \frac{d^{2} U_{\theta}}{d \Delta \theta^{2}}=k_{\theta}, \\
& U_{\tau}=U_{\phi}+U_{\omega}=\frac{1}{2} k_{\varphi}(\Delta \varphi)^{2}, \quad \frac{d^{2} U_{\tau}}{d \Delta \varphi^{2}}=k_{\varphi},
\end{aligned}
$$

where $k_{r}, k_{\theta}$, and $k_{\varphi}$ represent the bond stretching force constant, bond angle variation force constant, and out-ofplane angle variation resistance, respectively. In (9), the terms $\Delta r, \Delta \theta$, and $\Delta \varphi$ indicate the increase in bond length, bond angle change, and out-of-plane change, respectively. Equalization of the well-known structural energies with molecular energies in (9) leads to the following formulations [25]:

$$
\frac{E A}{L}=k_{r}, \quad \frac{E I}{L}=k_{\theta}, \quad \frac{G J}{L}=k_{\varphi},
$$

where $E$ and $G$ are the elastic modulus and shear modulus, and $A, L, I$, and $J$ are cross section area, length, moment of inertia, and polar moment of inertia of the beam element, respectively. After simplifications, the mechanical constants of the three-dimensional beam element with circular cross section having diameter $d$ are obtained as follows [25]:

$$
\begin{gathered}
d=4 \sqrt{\frac{k_{\theta}}{k_{r}}}, \quad E=\frac{k_{r}^{2} L}{4 \pi k_{\theta}}, \\
G=\frac{k_{r}^{2} k_{\varphi} L}{8 \pi k_{\theta}} .
\end{gathered}
$$

The values of the cross-sectional area, the area moment of inertia $\left(I_{y y}=I_{z z}\right)$, and the torsional moment of inertia $\left(I_{x x}\right)$ 


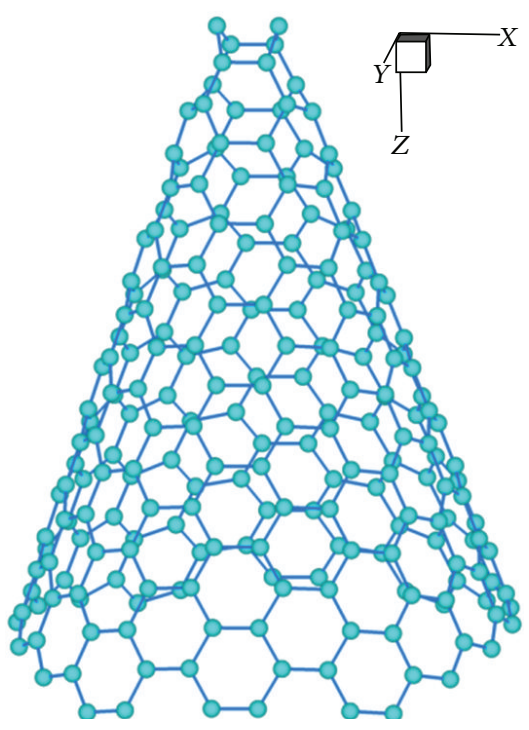

(a)

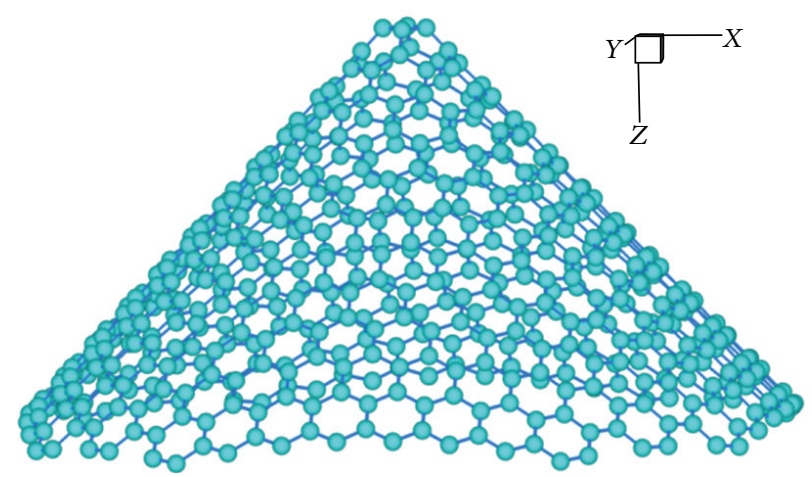

(c)

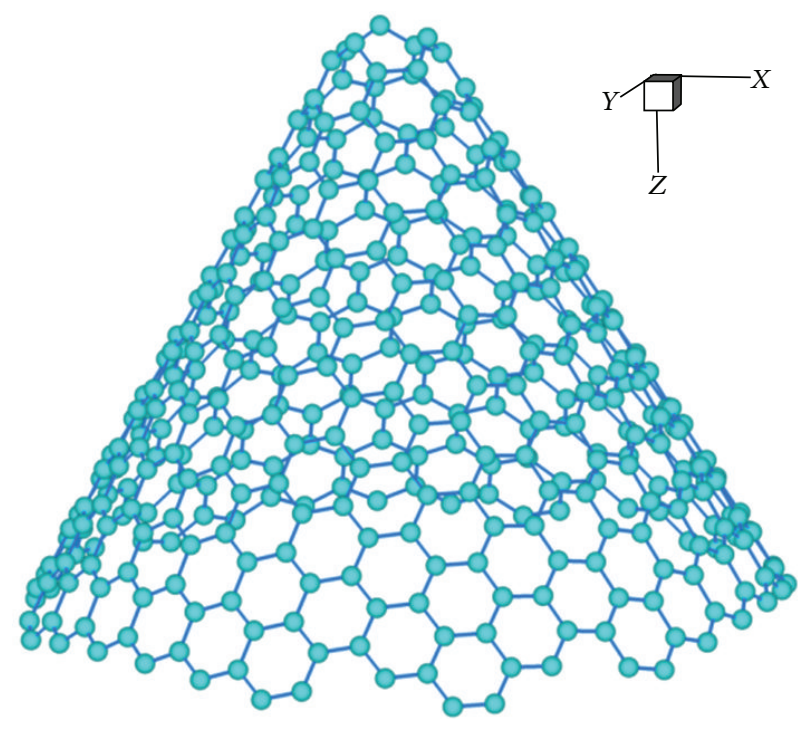

(b)

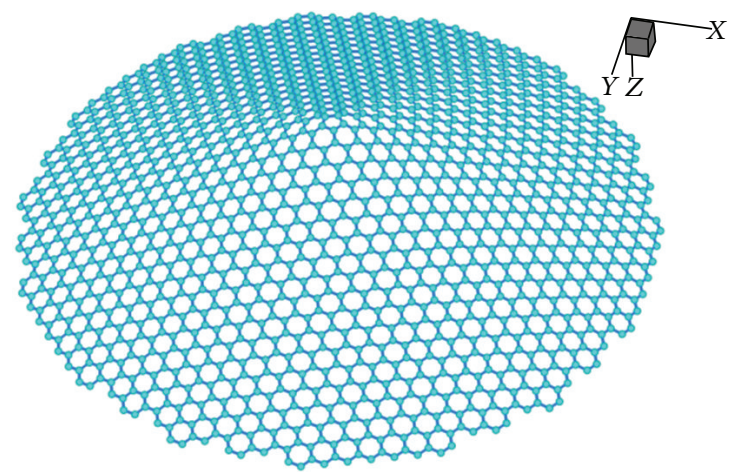

(d)

FIGURE 3: Cones with apex angles of (a) $38.9^{\circ}$, (b) $60^{\circ}$, (c) $86.6^{\circ}$, and (d) $123.6^{\circ}$, with cone heights of $20 \AA$.
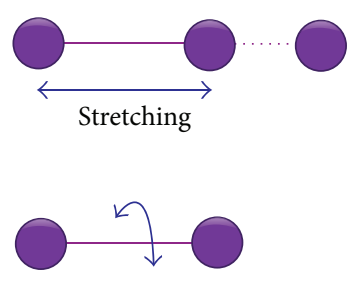

Dihedral angle torsion

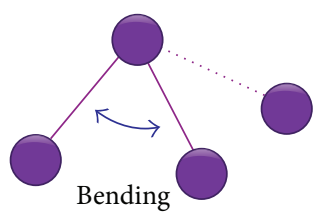

FIGURE 4: Various bonds between carbon atoms.

are considered to be $1.68794 \AA^{2}, 0.453456 \AA^{4}$, and $0.22682 \AA^{4}$, respectively, and the covalent bond distance of the carbon atoms is $0.142 \mathrm{~nm}$ [25]. Substitution of these values into (11) results in the following:

$$
\begin{gathered}
d=1.466 \AA, \quad E=5.488 \times 10^{-8} \mathrm{~N} / \AA^{2}, \\
G=8.701 \times 10^{-9} \mathrm{~N} / \AA^{2} .
\end{gathered}
$$

\section{Application of FEM in the Vibrational Analysis of the CNC}

In this section, the finite element model which is used to analyze the vibrational behavior of the CNCs is clarified. The FEM is performed using the commercial code ANSYS. The CNCs with different boundary conditions are modeled to obtain the natural frequencies and the corresponding mode shapes. As it was mentioned in Section 3, the covalent bonds between two carbon atoms are modeled using the beam element and each atom is considered as point mass element. The free vibration of a nanostructure can be formulated in the form of

$$
[M]\{\ddot{q}\}+[K]\{q\}=0,
$$




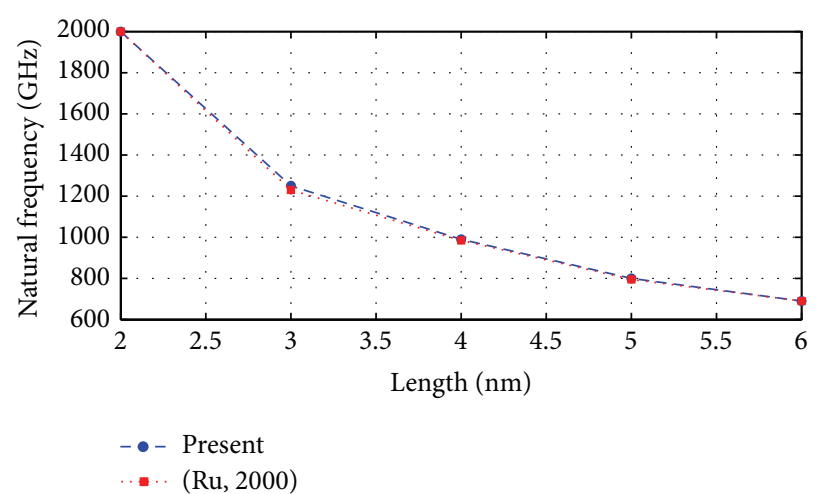

(a)

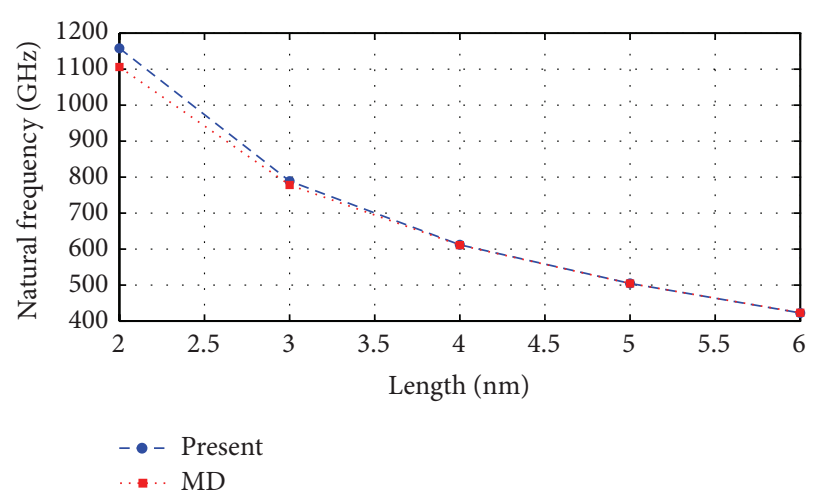

(b)

FIGURE 5: Comparison of the obtained natural frequency using BEAM4 and BEAM188 elements with the results of MD simulations and [10] (apex angle $=38.9^{\circ}$ ).

where $[M],[K],\{q\}$, and $\{\ddot{q}\}$ indicate the global mass matrix, global stiffness matrix, nodal displacement vector, and nodal acceleration vector, respectively. The aforementioned matrices should be assembled for the whole of a structure. For a linear system, the nodal displacement vector is considered as follows:

$$
\{q\}=\{\varphi\}_{i} \cos \omega_{i} t,
$$

in which $\omega_{i}$ is the $i$ th natural frequency, $\{\varphi\}_{i}$ is the vector that represents the mode shapes associated with $\omega_{i}$, and $t$ denotes the time variable. Substituting (14) into (13) leads to the following:

$$
\left([K]-\omega_{i}^{2}[M]\right)\{\varphi\}_{i}=\{0\},
$$

in which $\{\varphi\}_{i}$ is $i$ th mode shape. To obtain the natural frequencies, the determinant of the term in the parentheses in (15) must be equal to zero. By substitution of the natural frequencies into (15), the corresponding mode shapes are determined. The above finite element formulation and simulation were carried out using shifted Block Lanczos technique in ANSYS which is applicable for symmetric eigenvalue problems [37].

\section{Molecular Dynamics}

In order to assess the results generated by FEM for vibrational behavior of SWCNC, MD simulation is utilized herein. In this regard, the Tersoff-Brenner [38, 39] potential energy function is used to calculate energetics of carbon bonds within the canonical ensemble known as NVT at room temperature $(300 \mathrm{~K})$. This potential function is given by

$$
V\left(r_{i j}\right)=\left(\frac{1}{2}\right) f_{c}\left(r_{i j}\right)\left[V^{R}\left(r_{i j}\right)+b_{i j} V^{A}\left(r_{i j}\right)\right],
$$

where $r_{i j}$ expresses the distance between atoms $i$ and $j, V^{R}\left(r_{i j}\right)$ and $V^{A}\left(r_{i j}\right)$ stand for the repulsive and attractive pairwise interaction terms, respectively, and $b_{i j}$ is the strength of $V^{A}\left(r_{i j}\right)$.

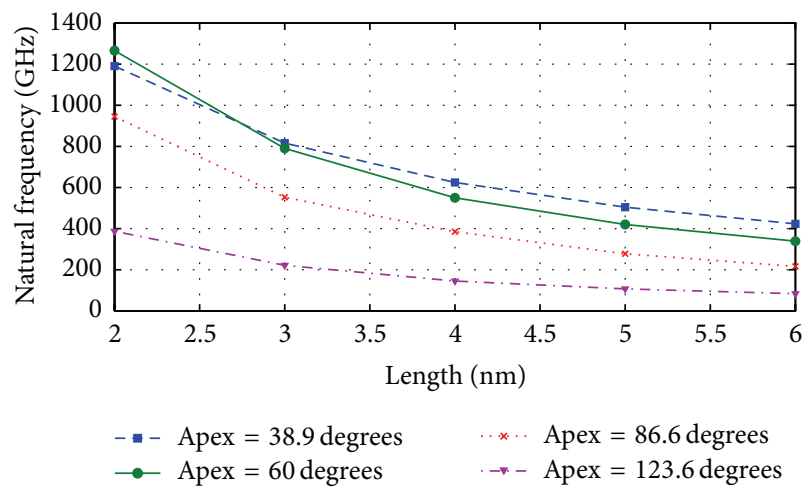

FIGURE 6: Variation of natural frequencies of the SWCNCs versus the length for different apex angles.

To integrate the equations of motion through time, Velocity-Verlet algorithm [40] is employed together with Nose-Hoover algorithm [41]. Moreover, to conserve energy and reducing temperature fluctuation and prevent system instability, the time step of $1 \mathrm{fs}$ is chosen. Further, to compute the natural frequency of SWCNC, the system initially was relaxed for 3 ps to reach its optimum state of energy; then after applying initial proper deflection similar to first mode shape [42], it is allowed to vibrate freely. Finally, using the time history of center of mass position during simulation, natural frequencies are computed by using the Fast Fourier Transform (FFT) method.

\section{Results and Discussions}

In this section, the numerical results are presented. To this end, SWCNCs are modeled using molecular structural model in which the $\mathrm{C}-\mathrm{C}$ bonds are simulated using beam elements. Besides, mass elements are used to model the carbon atoms located at the sides of beams. The MASS21 element with the concentrated mass of $1.9943 \times 10^{-23} \mathrm{~g}$ is placed at the corners of beam elements.

The usage of the present analysis for the vibration behavior of SWCNCs has been validated in Figure 5. This 


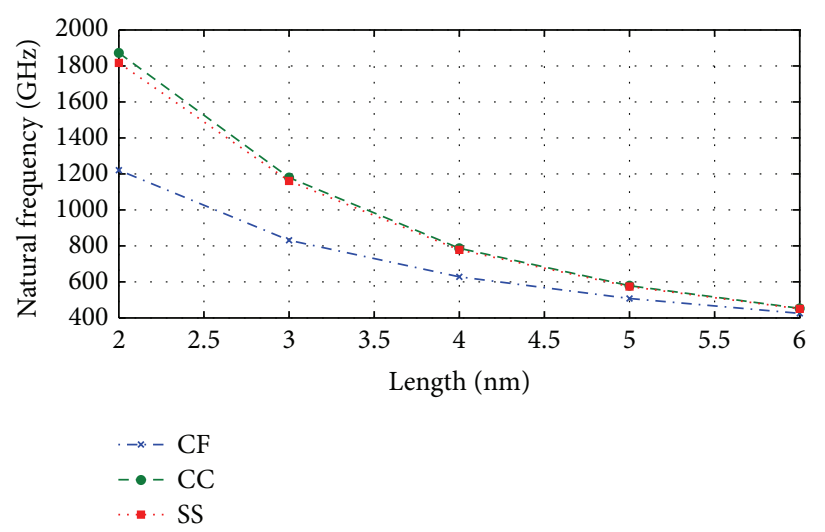

(a)

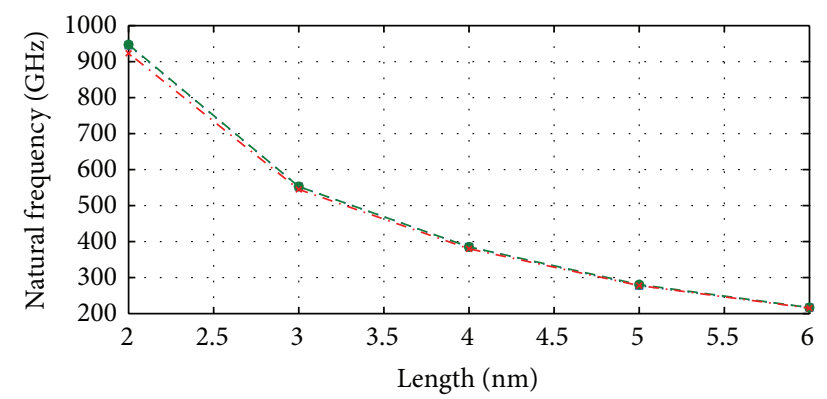

$\cdots$ CF
-- CC
$-x-S S$

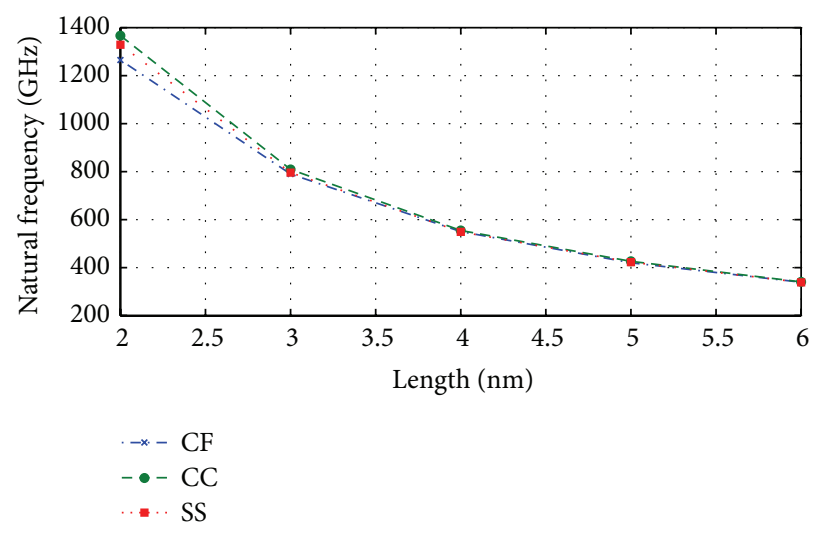

(b)

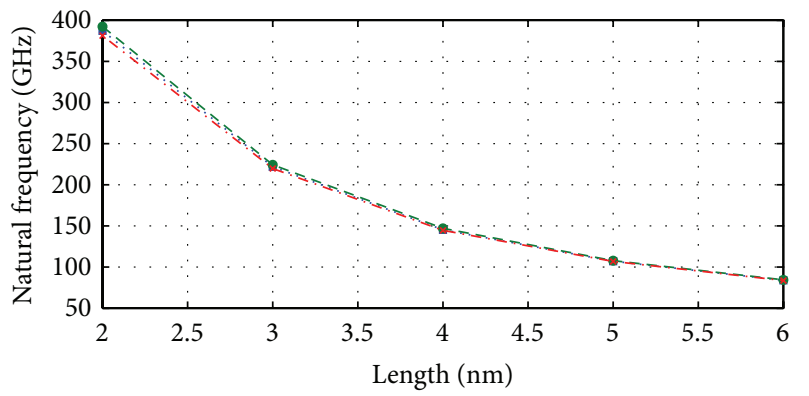

-... CF

$-\bullet$ CC

$-x-$ SS

(c)

(d)

FIGURE 7: Variation of natural frequencies of the SWCNCs with the length for different apex angles and various boundary conditions: (a) apex angle $=38.9^{\circ}$, (b) $60^{\circ}$, (c) $86.6^{\circ}$, and (d) $123.6^{\circ}$.

figure demonstrates the variation of the natural frequency of SWCNC with apex angle of $38.9^{\circ}$ versus the length of SWCNC. The three-dimensional elastic BEAM4 element is used to model the bonds which has six degrees of freedom (DOFs) in each node, including three translations in $x, y$, and $z$ directions and three rotations about these axes. As it is observed, the present numerical results meet an excellent agreement with those reported in [35]. It is clear that by increasing the length of the nanocone, natural frequency decreases and this fact can be generalized to all of the possible apex angles.

To make a comparison between the results of the present study and those predicted by MD simulation, the threedimensional elastic BEAM188 element is also utilized for the modeling of the bonds. The utilized beam theory for BEAM188 element is based on the Timoshenko beam model, which is a first-order shear deformation theory; but for BEAM4 element, the shear deformation is neglected [43]. The values of Young's modulus, Poisson's ratio, and cross section radius are given as the input of this element. These values are given in (12). Figure 5 also shows that the obtained results by using BEAM188 element are in excellent agreement with those generated from MD simulation and it implies the accuracy of the present method. Comparing the given curves in Figure 5, one can conclude that, to have rational results which can trace result of $\mathrm{MD}$ simulations, the element BEAM188 should be utilized. So, in all of the subsequent results, the BEAM188 element is used to simulate the C-C bonds.

Figure 6 gives the variation of natural frequency of the SWCNCs with different apex angles versus the SWCNC length. It can be found that, by increasing the apex angle of SWCNCs, the value of natural frequency decreases. It is also seen that increasing apex angle results in decreasing the sensitivity of vibrational behavior to SWCNC length. Besides, increasing side length results in decreasing the sensitivity to length change.

The variations of the natural frequencies of the SWCNCs with apex angles of $38.9^{\circ}, 60^{\circ}, 86.6^{\circ}$, and $123.6^{\circ}$ for different boundary conditions are illustrated in Figure 7 versus the SWCNC length. Three different boundary conditions are considered including clamped-clamped (CC), clamped-free (CF), and simply supported-simply supported (SS). It should be noted that all of the DOFs of the nodes located at the boundary are constrained for clamped boundary conditions. For simply supported ones, only translational DOFs are constrained and the boundary nodes can revolve in rotational DOFs. The natural frequencies of the SWCNCs with CC 


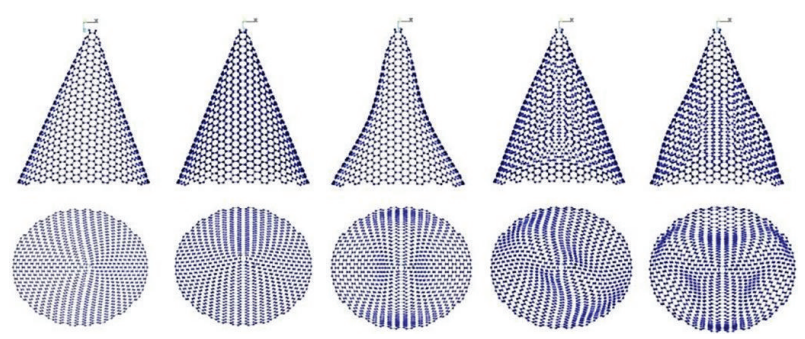

(a)
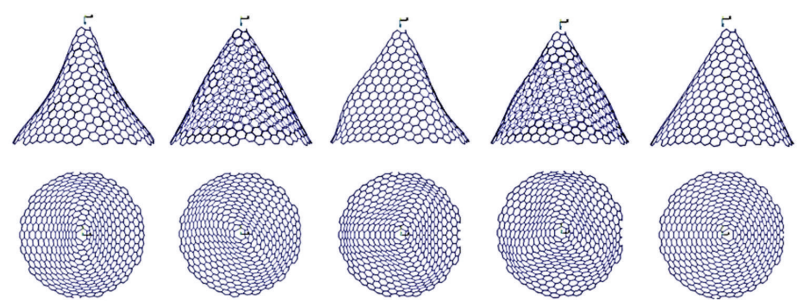

(b)

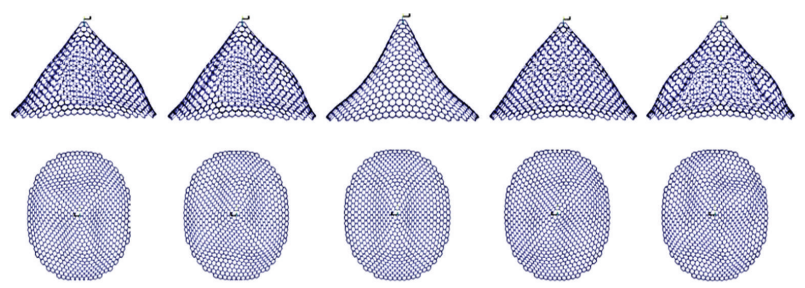

(c)

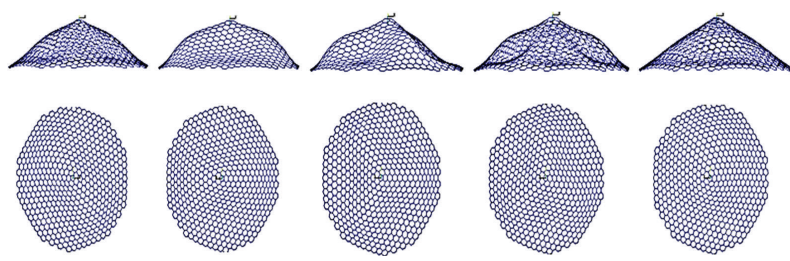

(d)

FIGURE 8: First five mode shapes of the SWCNC with various apex angles: (a) apex angle $=38.9^{\circ}$, (b) $60^{\circ}$, (c) $86.6^{\circ}$, and (d) $123.6^{\circ}$.

boundary conditions are higher than those with the two other end supports. However, in higher apex angles, the dependency of natural frequencies on the boundary conditions decreases. Finally, the first five mode shapes of SWCNCs with various apex angles are shown in Figure 8. It is seen that SWCNCs with different apex angles experience different mode shapes. So, one can conclude that the mode shapes of SWCNCs depend on their apex angles.

\section{Conclusion}

In this paper, the vibrational behavior of SWCNCs with different lengths and apex angles was studied. Besides, the SWCNCs under various boundary conditions were examined. To this end, a molecular structural model was used. Comparing the results of the model with those of $\mathrm{MD}$ simulations, it was shown that modeling $\mathrm{C}-\mathrm{C}$ bonds by BEAM188 element can accurately predict the fundamental natural frequencies of SWCNCs. It was shown that increasing side length of a SWCNC with a constant apex angle results in decreasing the frequency. Moreover, it was seen that the SWCNCs with larger apex angles have smaller frequencies. Finally, comparing the frequencies of SWCNCs under different boundary conditions, it was observed that increasing apex angle leads to decreasing the sensitivity of vibrational behavior of SWCNCs to their end conditions.

\section{Conflict of Interests}

The authors declare that there is no conflict of interests regarding to the publication of this paper.

\section{References}

[1] S. Iijima, "Helical microtubules of graphitic carbon," Nature, vol. 354 , no. 6348, pp. 56-58, 1991.

[2] H. W. Kroto, J. R. Heath, S. C. O'Brien, R. F. Curl, and R. E. Smalley, " $\mathrm{C}_{60}$ : Buckminsterfullerene," Nature, vol. 318, no. 6042, pp. 162-163, 1985.

[3] X. Y. Kong, Y. Ding, R. Yang, and Z. L. Wang, "Single-crystal nanorings formed by epitaxial self-coiling of polar nanobelts," Science, vol. 303, no. 5662, pp. 1348-1351, 2004.

[4] S. Lijima, T. Ichihashi, and Y. Ando, "Pentagons, heptagons and negative curvature in graphite microtubule growth," Nature, vol. 356, no. 6372, pp. 776-778, 1992.

[5] S. Iijima and T. Ichihashi, "Single-shell carbon nanotubes of 1nm diameter," Nature, vol. 363, no. 6430, pp. 603-605, 1993.

[6] S.-S. Yu and W.-T. Zheng, "Effect of N/B doping on the electronic and field emission properties for carbon nanotubes, carbon nanocones, and graphene nanoribbons," Nanoscale, vol. 2, no. 7, pp. 1069-1082, 2010.

[7] R. Majidi and K. Ghafoori Tabrizi, "Study of neon adsorption on carbon nanocones using molecular dynamics simulation," Physica B: Condensed Matter, vol. 405, no. 8, pp. 2144-2148, 2010.

[8] Y. Hu, K. M. Liew, X. Q. He, Z. Li, and J. Han, "Free transverse vibration of single-walled carbon nanocones," Carbon, vol. 50, no. 12, pp. 4418-4423, 2012.

[9] J. W. Yan, K. M. Liew, and L. H. He, "Ultra-sensitive analysis of a cantilevered single-walled carbon nanocone-based mass detector," Nanotechnology, vol. 24, no. 12, Article ID 125703, 2013.

[10] C. Q. Ru, "Effective bending stiffness of carbon nanotubes," Physical Review B: Condensed Matter and Materials Physics, vol. 62, no. 15, pp. 9973-9976, 2000.

[11] A. Krishnan, E. Dujardin, M. M. J. Treacy, J. Hugdahl, S. Lynum, and T. W. Ebbesen, "Graphitic cones and the nucleation of curved carbon surfaces,” Nature, vol. 388, no. 6641, pp. 451-454, 1997.

[12] S. N. Naess, A. Elgsaeter, G. Helgesen, and K. D. Knudsen, "Carbon nanocones: wall structure and morphology," Science and Technology of Advanced Materials, vol. 10, no. 6, Article ID 065002, 2009.

[13] S. Compernolle, B. Kiran, L. F. Chibotaru, M. T. Nguyen, and A. Ceulemans, "Ab initio study of small graphitic cones with triangle, square, and pentagon apex," Journal of Chemical Physics, vol. 121, no. 5, pp. 2326-2336, 2004.

[14] S. Iijima, C. Brabec, A. Maiti, and J. Bernholc, "Structural flexibility of carbon nanotubes," Journal of Chemical Physics, vol. 104, no. 5, pp. 2089-2092, 1996. 
[15] B. I. Yakobson, M. P. Campbell, C. J. Brabec, and J. Bernholc, "High strain rate fracture and C-chain unraveling in carbon nanotubes," Computational Materials Science, vol. 8, no. 4, pp. 341-348, 1997.

[16] D. Sánchez-Portal, E. Artacho, J. M. Soler, A. Rubio, and P. Ordejón, "Ab initio structural, elastic, and vibrational properties of carbon nanotubes," Physical Review B-Condensed Matter and Materials Physics, vol. 59, no. 19, pp. 12678-12688, 1999.

[17] C. M. Wang, V. B. C. Tan, and Y. Y. Zhang, "Timoshenko beam model for vibration analysis of multi-walled carbon nanotubes," Journal of Sound and Vibration, vol. 294, no. 4, pp. 1060-1072, 2006.

[18] J.-C. Hsu, R.-P. Chang, and W.-J. Chang, "Resonance frequency of chiral single-walled carbon nanotubes using Timoshenko beam theory," Physics Letters A, vol. 372, no. 16, pp. 2757-2759, 2008.

[19] Y. Y. Zhang, C. M. Wang, and V. B. C. Tan, "Assessment of Timoshenko beam models for vibrational behavior of singlewalled carbon nanotubes using molecular dynamics," Advances in Applied Mathematics and Mechanics, vol. 1, no. 1, pp. 89-106, 2009.

[20] B. I. Yakobson, C. J. Brabec, and J. Bernholc, "Nanomechanics of carbon tubes: instabilities beyond linear response," Physical Review Letters, vol. 76, no. 14, pp. 2511-2514, 1996.

[21] C. Q. Ru, "Elastic buckling of single-walled carbon nanotube ropes under high pressure," Physical Review B, vol. 62, no. 15, pp. 10405-10408, 2000.

[22] G. M. Odegard, T. S. Gates, L. M. Nicholson, and K. E. Wise, "Equivalent-continuum modeling of nano-structured materials," Composites Science and Technology, vol. 62, no. 14, pp. 18691880, 2002.

[23] C. Li and T. Chou, "A structural mechanics approach for the analysis of carbon nanotubes," International Journal of Solids and Structures, vol. 40, no. 10, pp. 2487-2499, 2003.

[24] S. Rouhi and R. Ansari, "Atomistic finite element model for axial buckling and vibration analysis of single-layered graphene sheets," Physica E, vol. 44, no. 4, pp. 764-772, 2012.

[25] R. Ansari and S. Rouhi, "Atomistic finite element model for axial buckling of single-walled carbon nanotubes," Physica E: LowDimensional Systems and Nanostructures, vol. 43, no. 1, pp. 5869, 2010.

[26] K. M. Liew, Z. X. Lei, J. L. Yu, and L. W. Zhang, "Postbuckling of carbon nanotube-reinforced functionally graded cylindrical panels under axial compression using a meshless approach," Computer Methods in Applied Mechanics and Engineering, vol. 268, pp. 1-17, 2014.

[27] L. W. Zhang, Z. X. Lei, K. M. Liew, and J. L. Yu, "Static and dynamic of carbon nanotube reinforced functionally graded cylindrical panels," Composite Structures, vol. 111, pp. 205-212, 2014.

[28] K. M. Liew, Z. X. Lei, J. L. Yu, and L. W. Zhang, "Postbuckling of carbon nanotube-reinforced functionally graded cylindrical panels under axial compression using a meshless approach," Computer Methods in Applied Mechanics and Engineering, vol. 268, pp. 1-17, 2014.

[29] J. X. Wei, K. M. Liew, and X. Q. He, "Mechanical properties of carbon nanocones," Applied Physics Letters, vol. 91, no. 26, Article ID 261906, 2007.

[30] K. M. Liew, J. X. Wei, and X. Q. He, "Electronic structure: wideband, narrow-band, and strongly correlated systems," Physical Review B, vol. 75, no. 19, article 1, 2007.
[31] R. D. Firouz-Abadi, M. M. Fotouhi, and H. Haddadpour, "Free vibration analysis of nanocones using a nonlocal continuum model," Physics Letters A: General, Atomic and Solid State Physics, vol. 375, no. 41, pp. 3593-3598, 2011.

[32] S. P. Jordan and V. H. Crespi, "Theory of carbon nanocones: mechanical chiral inversion of a micron-scale threedimensional object," Physical Review Letters, vol. 93, Article ID 255504, 4 pages, 2004.

[33] J. W. Yan, D. Li, K. M. Liew, and L. H. He, "Predicting mechanical properties of single-walled carbon nanocones using a higher-order gradient continuum computational framework," Composite Structures, vol. 94, no. 11, pp. 3271-3277, 2012.

[34] J. H. Lee and B. S. Lee, "Modal analysis of carbon nanotubes and nanocones using FEM," Computational Materials Science, vol. 51, no. 1, pp. 30-42, 2012.

[35] M. M. Seyyed Fakhrabadi, N. Khani, and S. Pedrammehr, "Vibrational analysis of single-walled carbon nanocones using molecular mechanics approach," Physica E: Low-Dimensional Systems and Nanostructures, vol. 44, no. 7-8, pp. 1162-1168, 2012.

[36] M. M. S. Fakhrabadi, N. Khani, R. Omidvar, and A. Rastgoo, "Investigation of elastic and buckling properties of carbon nanocones using molecular mechanics approach," Computational Materials Science, vol. 61, pp. 248-256, 2012.

[37] R. G. Grimes, J. G. Lewis, and H. D. Simon, "A shifted block Lanczos algorithm for solving sparse symmetric generalized eigenproblems," SIAM Journal on Matrix Analysis and Applications, vol. 15, no. 1, pp. 228-272, 1994.

[38] J. Tersoff, "New empirical approach for the structure and energy of covalent systems," Physical Review B, vol. 37, no. 12, pp. 6991$7000,1988$.

[39] D. W. Brenner, "Empirical potential for hydrocarbons for use in simulating the chemical vapor deposition of diamond films," Physical Review B, vol. 42, no. 15, pp. 9458-9471, 1990.

[40] M. P. Allen and D. J. Tildesley, Computer Simulation of Liquids, Oxford University Press, New York, NY, USA, 1989.

[41] W. G. Hoover, "Canonical dynamics: Equilibrium phase-space distributions," Physical Review A, vol. 31, no. 3, pp. 1695-1697, 1985.

[42] R. Ansari, S. Ajori, and B. Arash, "Vibrations of single- and double-walled carbon nanotubes with layerwise boundary conditions: a molecular dynamics study," Current Applied Physics, vol. 12, no. 3, pp. 707-711, 2012.

[43] Element Library, Element Reference, Ansys Help Guide, Ver. 14, 2011. 

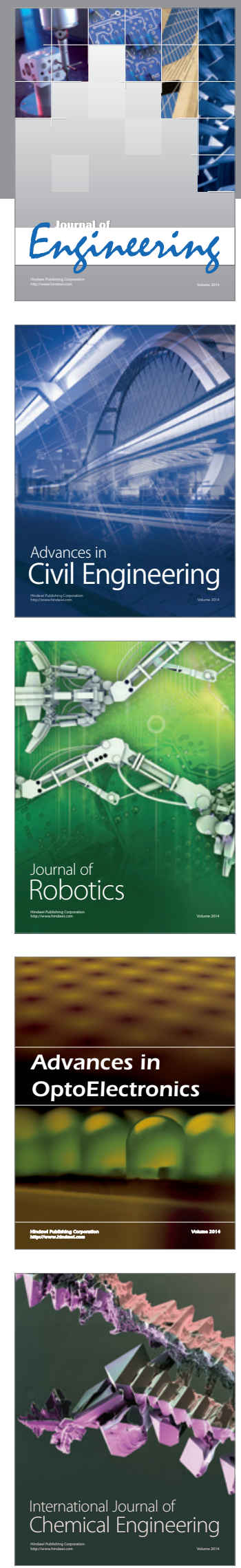

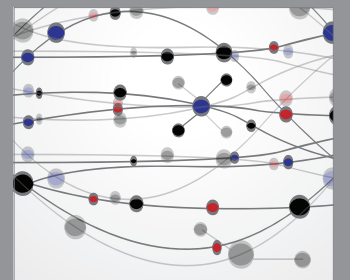

The Scientific World Journal
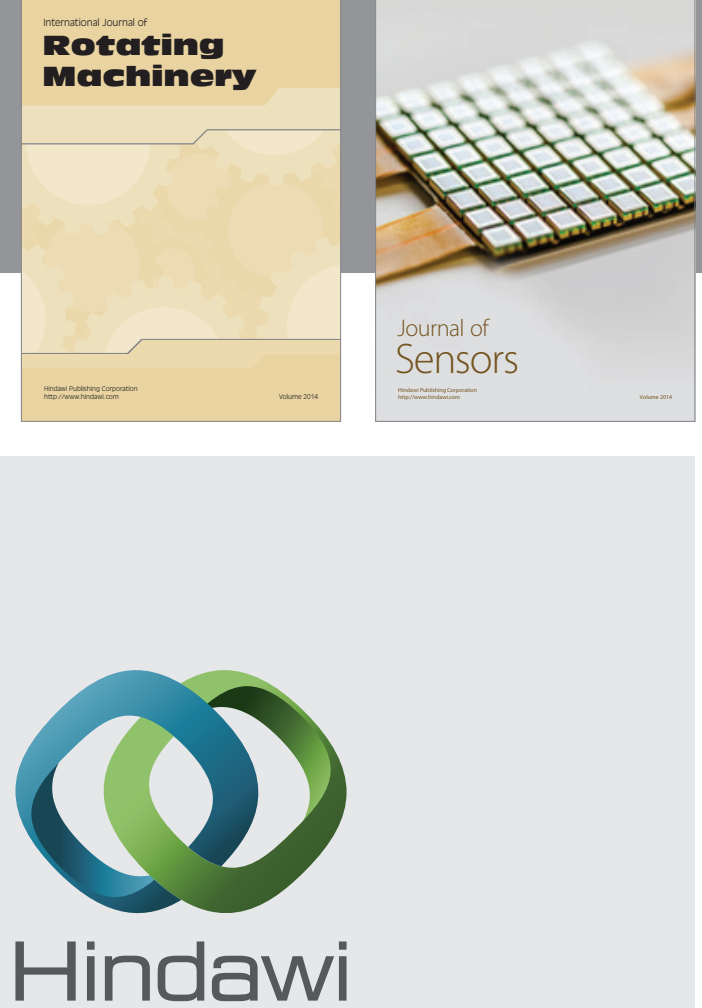

Submit your manuscripts at http://www.hindawi.com
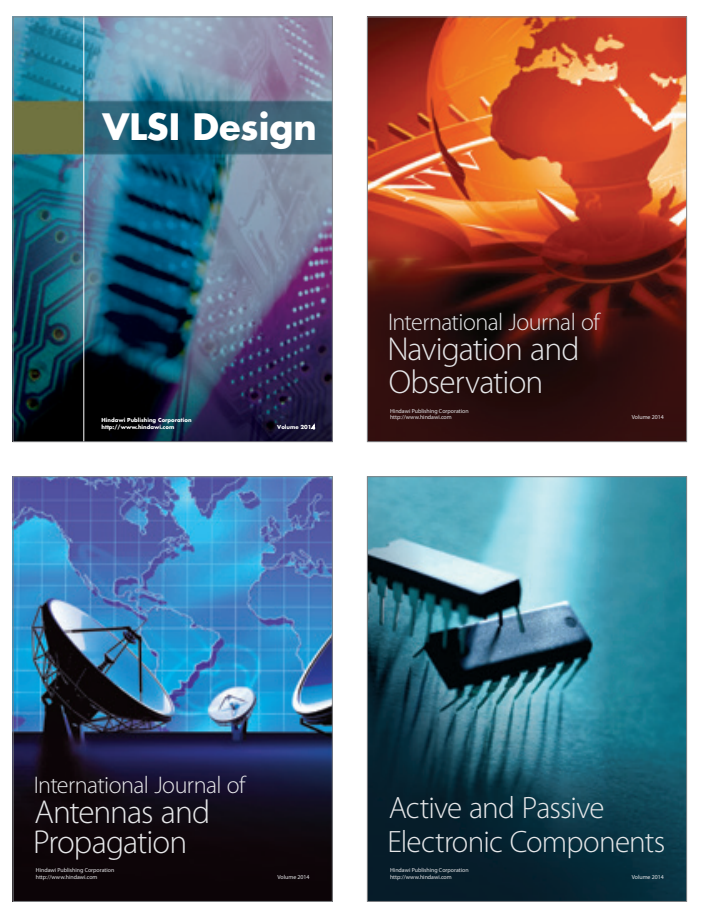
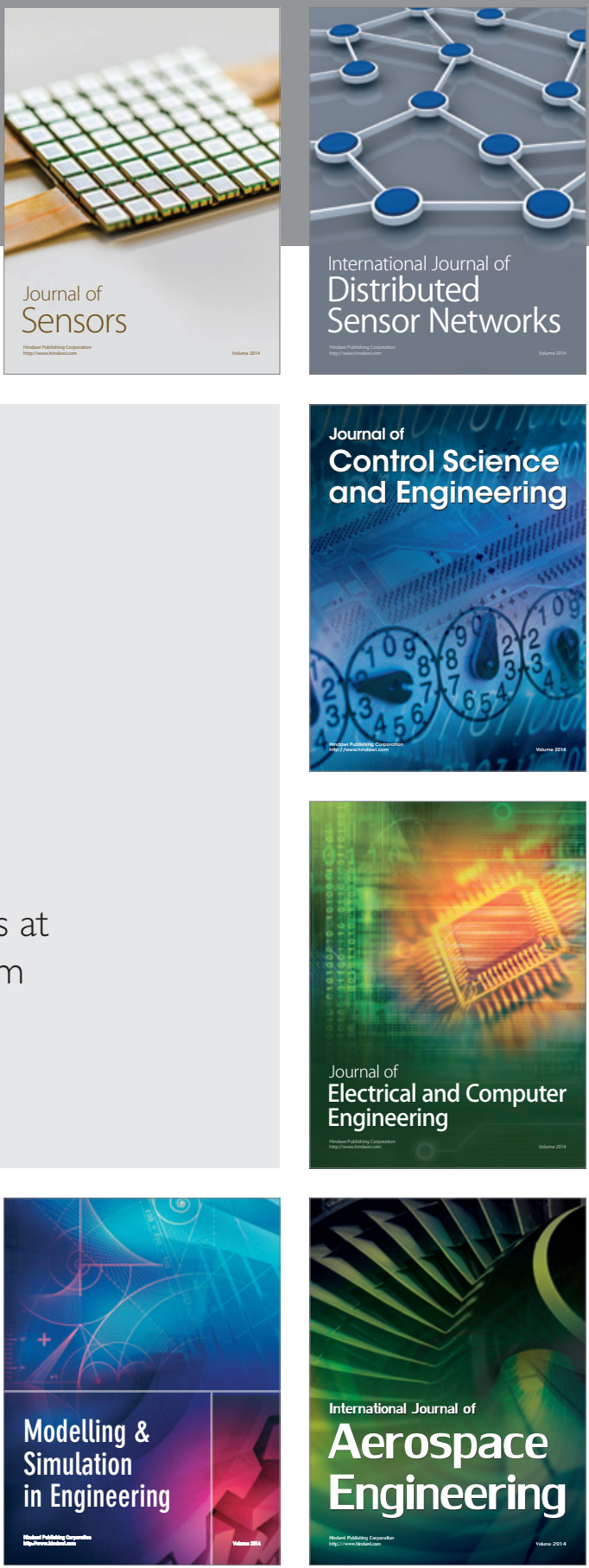

Journal of

Control Science

and Engineering
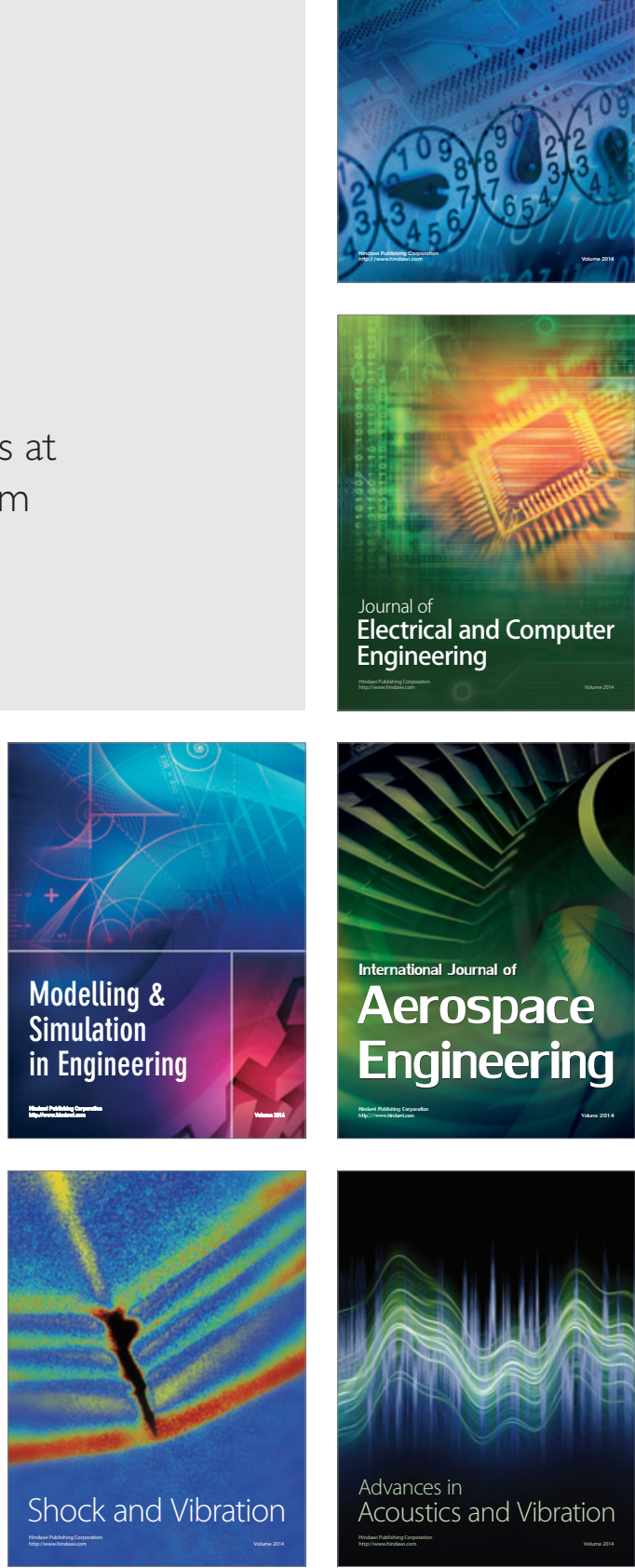